\title{
Endoscopic ultrasound-guided pancreatic duct stent placement for symptomatic pancreaticojejunostomy stricture
}

A 57-year-old woman underwent a pancreaticoduodenectomy for a duct-type intraductal papillary mucinous neoplasm in the pancreatic head. She presented 3 years later with recurrent episodes of acute pancreatitis, with computed tomography (CT) revealing swelling of the pancreas remnant and dilation of the pancreatic duct, suggesting pancreaticojejunostomy (PJS) stricture. Endoscopic ultrasound (EUS)-guided pancreatic duct drainage was considered as a viable option for patient management ( $\vee$ Video 1 ). We advanced the echoendoscope into the stomach and identified the $4.6-\mathrm{mm}$ main pancreatic duct ( Fig.1). We punctured the pancreatic duct with a 19-gauge flexible needle and performed pancreatography ( $\triangleright$ Fig. 2). A 0.025-inch guidewire could not be negotiated across the PJS anastomosis (- Fig.3). Next, we inserted a 6-Fr cystotome followed by the deployment of a $5 \mathrm{Fr} \times 7 \mathrm{~cm}$ plastic stent; the proximal extremity of the stent was positioned in the pancreatic duct and the distal end was in the gastric lumen ( $\triangleright$ Fig. 4 ).

The recovery was uneventful and the patient was discharged 5 days after the procedure. A CT scan 4 months later demonstrated the good position of the pancreatic stent ( $>$ Fig.5). In the following 6 months, acute pancreatitis did not recur, and amylase and lipase blood levels returned to normal.

The incidence of long-term PJS stricture following pancreaticoduodenectomy resection for benign disease ranges from $5 \%$ to $10 \%[1,2]$. Surgical reintervention for this adverse event is complex [3]. Therefore, many authors favor less invasive initial approaches, namely enteroscopy-assisted endoscopic retrograde pancreatography (e-ERP).

EUS-guided pancreatic duct drainage has advanced significantly and provides an alternative to e-ERP. In an international multicenter retrospective study, EUS-

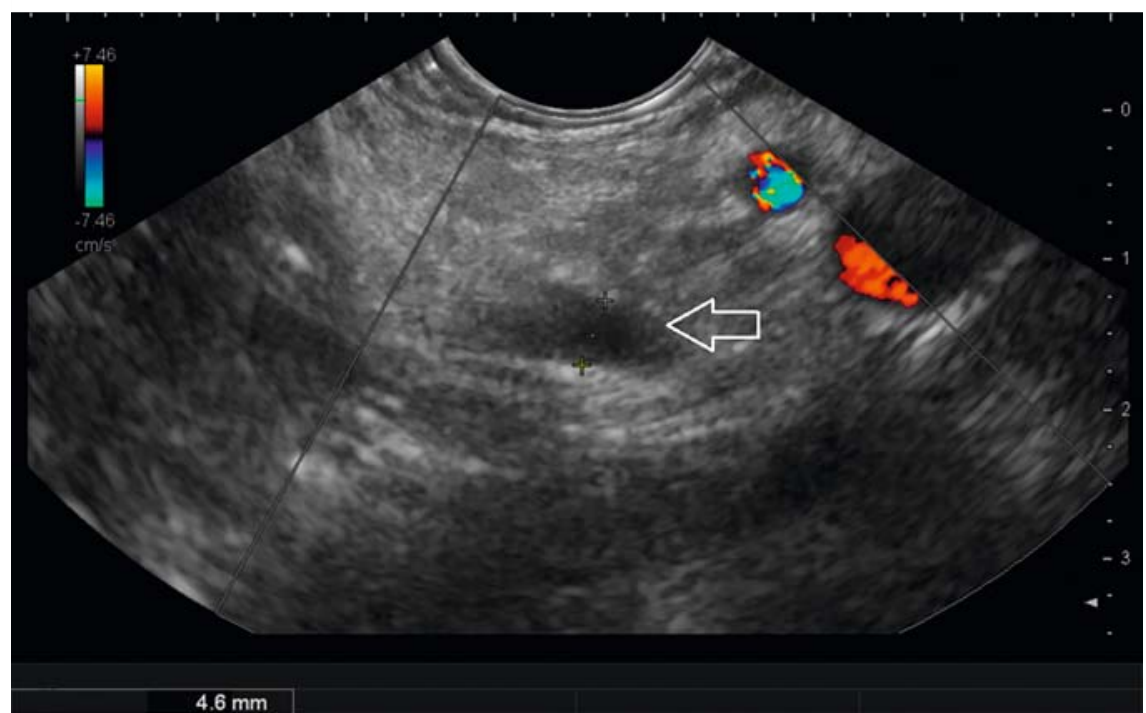

- Fig. 1 Endoscopic ultrasound view showing a dilated pancreatic duct (arrow).

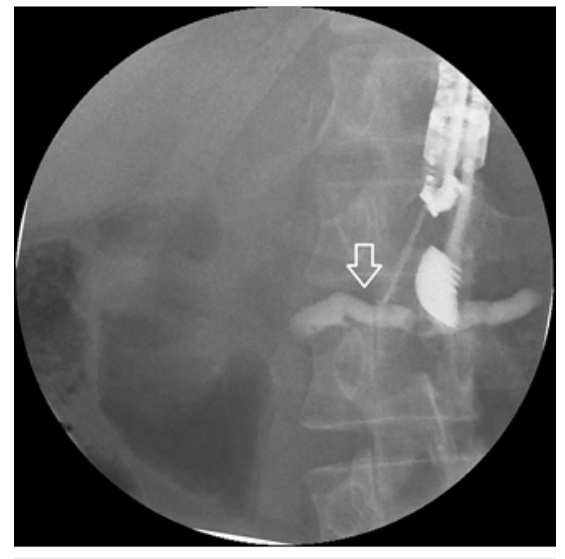

-Fig. 2 Endoscopic ultrasound-guided pancreatic drainage performed with a 19-gauge needle (arrow).

guided pancreatic duct drainage demonstrated better clinical success $(87.5 \%$ vs. $23.1 \%)$ but had a higher mild-to-moderate adverse event rate (35\% vs. 2.9\%) [4]. In conclusion EUS-guided pancreatic duct stent placement is technically challenging but a viable option to treat PJS stricture in symptomatic patients.

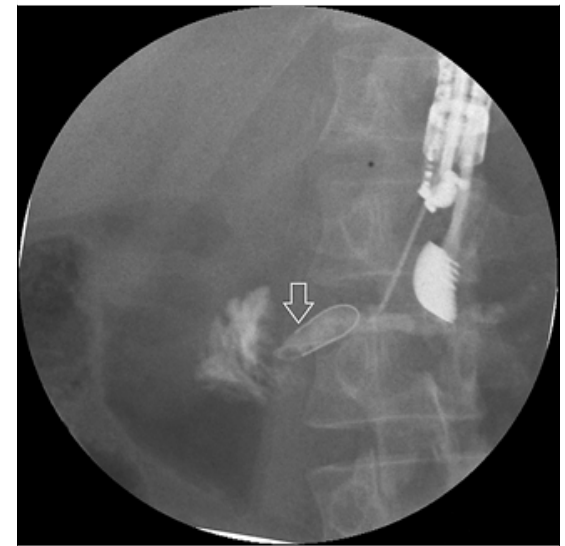

- Fig. 3 Attempt to negotiate a 0.025 inch guidewire across the stricture (arrow).

Endoscopy_UCTN_Code_TTT_1AS_2AD

Competing interests

None 


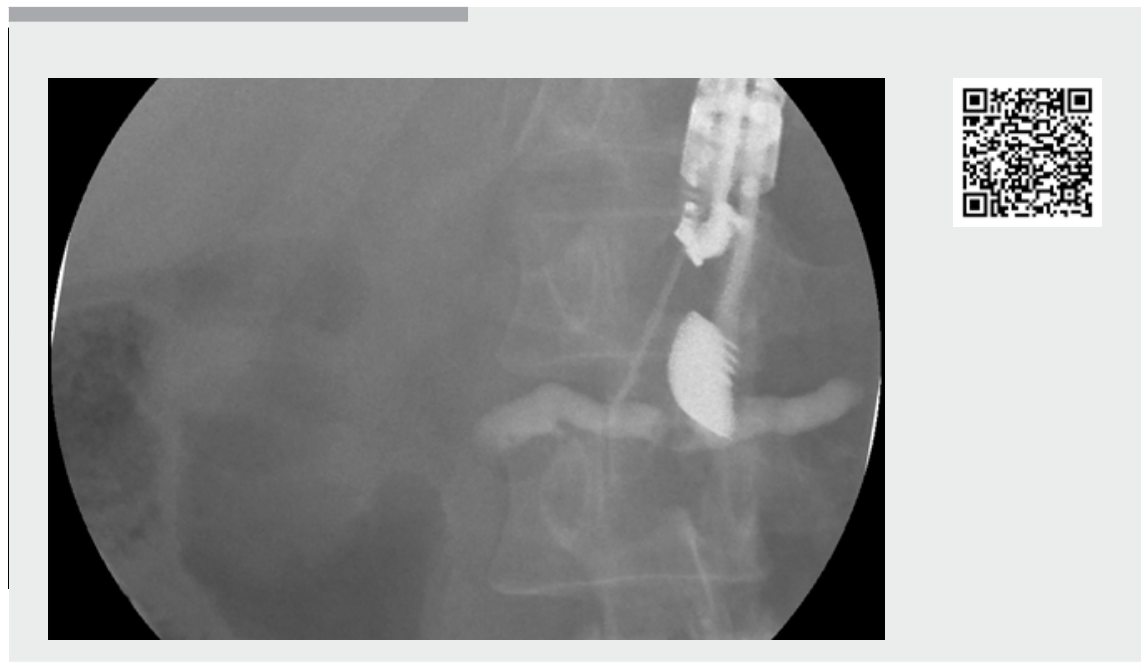

$\checkmark$ Video 1 Endoscopic ultrasound-guided pancreatic duct stent placement for pancreaticojejunostomy stricture.

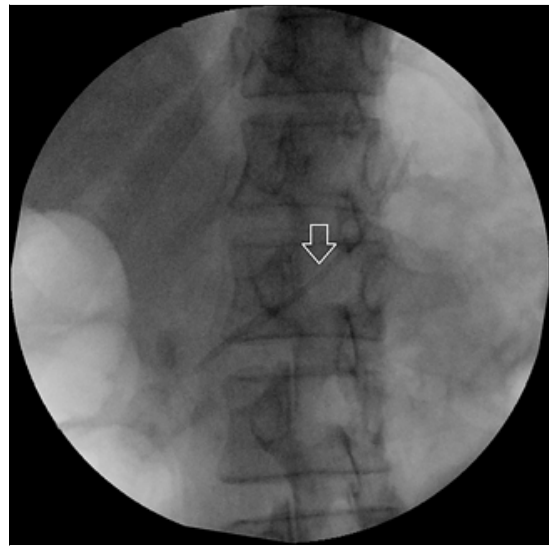

- Fig. 4 Placement of a 5-Fr plastic stent (arrow) from the stomach into the pancreatic duct.

The authors

\section{Joel Fernandez de Oliveira, Karen Costa}

\section{Carvalho Gon, Fauze Maluf-Filho}

Division of Endoscopy, Hospital Beneficência

Portuguesa, São Paulo, Brazil

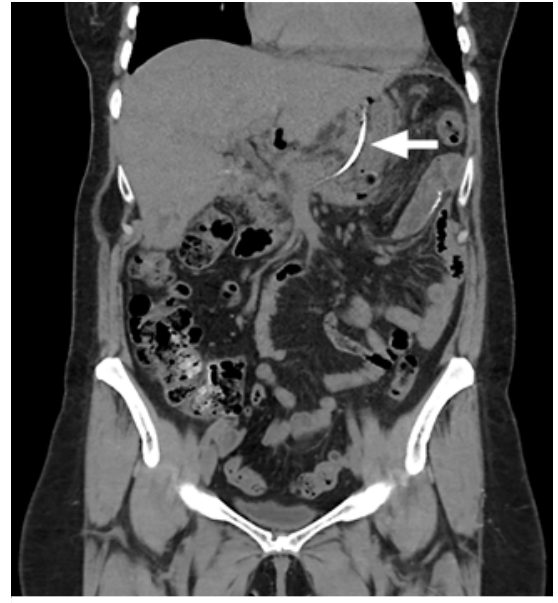

- Fig. 5 Computed tomography control demonstrated pancreatic stent (arrow) in

\section{Corresponding author}

\section{Joel Fernandez de Oliveira, MD, MSc}

Division of Endoscopy, Hospital Beneficência Portuguesa, Rua Maestro Cardim 769,

São Paulo, SP, 01323-900, Brazil

Fax: +55-11-35051000

jfoliveira1@gmail.com a good position.

[1] Reid-Lombardo KM, Ramos-De la Medina A, Thomsen K. Long-term anastomotic complications after pancreaticoduodenectomy for benign diseases. J Gastrointest Surg 2007; 11: $1704-1711$

[2] Morgan KA, Fontenot BB, Harvey NR et al. Revision of anastomotic stenosis after pancreatic head resection for chronic pancreatitis: is it futile? HPB (Oxford) 2010; 12 : $211-216$

[3] Krafft MR, Nasr JY. Anterograde endoscopic ultrasound-guided pancreatic duct drainage: a technical review. Dig Dis Sci 2019; 64: $1770-1781$

[4] Chen Yl, Levy MJ, Moreels TG et al. An international multicenter study comparing EUSguided pancreatic duct drainage with enteroscopy-assisted endoscopic retrograde pancreatography after Whipple surgery. Gastrointest Endosc 2017; 85: 170 - 177

\section{Bibliography}

DOI https://doi.org/10.1055/a-0999-5120

Published online: 17.9.2019

Endoscopy 2020; 52: E66-E67

(c) Georg Thieme Verlag KG

Stuttgart · New York

ISSN 0013-726X

\section{ENDOSCOPY E-VIDEOS}

https://eref.thieme.de/e-videos

回回 Endoscopy E-Videos is a free access online section, reporting 回然: on interesting cases and new

techniques in gastroenterological endoscopy. All papers include a high quality video and all contributions are freely accessible online.

This section has its own submission website at https://mc.manuscriptcentral.com/e-videos 